\title{
A Comprehensive Evaluation Between Dexmedetomidine and Midazolam for Intraoperative Sedation in the Elderly: Protocol for a Systematic Review and Meta-analysis of Randomized Controlled Trials
}

\section{Chunxia Huang}

Guangzhou University of Chinese Medicine

\section{Zunjiang Li}

Guangzhou University of Traditional Chinese Medicine: Guangzhou University of Chinese Medicine

Yingxin Long

Guangzhou University of Traditional Chinese Medicine: Guangzhou University of Chinese Medicine

Dongli Li

Guangzhou University of Traditional Chinese Medicine: Guangzhou University of Chinese Medicine

Manhua Huang

Guangzhou University of Traditional Chinese Medicine: Guangzhou University of Chinese Medicine

\section{Banghan Ding}

Guangdong Provincial Hospital of Traditional Chinese Medicine

Wei Zhu ( $013826260112 @ 163 . c o m)$

2nd Clinical Hospital of Guangzhou University of Chinese Medicine: Guangdong Provincial Hospital of Traditional Chinese Medicine https://orcid.org/0000-0003-1165-634X

\section{Protocol}

Keywords: Dexmedetomidine, Midazolam, meta-analysis, Intraoperative sedation, Randomized controlled trials

Posted Date: September 14th, 2021

DOI: https://doi.org/10.21203/rs.3.rs-864588/v1

License: (c) (i) This work is licensed under a Creative Commons Attribution 4.0 International License. Read Full License 


\section{Abstract}

Background: The sedative effect of intraoperative sedation in elderly surgery exerts critical influence on the prognosis. Comparison on the safety and efficacy between Dexmedetomidine and Midazolam in many clinical randomized controlled trials (RCTs) were inconsistent and suspicious. We aimed to comprehensively evaluate the safety and efficacy between Dexmedetomidine and Midazolam for intraoperative sedation in the elderly via meta-analysis and systematic reviews.

Methods: RCTs regarding to the comparison of sedative effects and safety between Dexmedetomidine and Midazolam in elderly patients (aged $\geq$ 60 years) will be comprehensively searched from 2000.10 to 2021.05 through 4 English databases and 4 Chinese databases. After extraction in duplicate, the systematic review and meta-analysis will be performed on the primary outcomes (hemodynamic changes, sedative effect, cognitive function) and secondary outcomes (analgesic effect, surgical characteristics, complications or adverse reactions) for assessing the two therapy methods using Review manage software (Version 5.3). Sensitivity analysis will be conducted to evaluate the heterogeneity of the results, funnel plot and Egger's test will be performed to analyze publication bias of the included studies, and test sequential analysis will be applied to assess the robustness and reliability of preliminary meta-analysis results. Finally, rating quality of evidence and strength of recommendations on the meta results will be summarized by rating quality of evidence and strength of recommendations (GRADE) approach.

Discussion: This systematic review and meta-analysis will evaluate the safety and efficacy between Dexmedetomidine and Midazolam for intraoperative sedation in the elderly, it will give an insight on the application of Dexmedetomidine and Midazolam, and will provide evidencesbased reference for clinical decision makings.

Systematic review registration: PROSPERO (CRD42021221897).

\section{Background}

In aging society, elderly population (aged $\geq 60$ years) suffers from increased risk of diseases due to their reduced physical function and weakened immune function, and they are increasingly dependent on surgical treatment[1, 2]. Older people have surgery at a higher rate than other age groups, making up $15 \%$ of the total population[3]. Despite significant advances in surgical anesthesia techniques and perioperative care, anesthetics are important cause of postoperative complications in the elderly[4], and comorbidities are consistently identified as major predictors of poor postoperative prognosis in this population[ $[5,6]$, thus the better and rational anesthetic medication in elderly surgery attracts increasing concern and interest.

In order to reduce the occurrence of postoperative complications, safe and effective anesthetics are issues that we need to consider in clinic. Dexmedetomidine is a highly selective a2-adrenergic receptor agonist, its sedation and analgesic effects are similar to opioids, and are suitable for short-term or long-term sedation in the intensive care environment[7-9]. Dexmedetomidine induces a dose-dependent effect, but this sedation is reversible, patient is easily awakened to a waking state[10-12], it is reported that Dexmedetomidine application may result in postoperative comorbidities including uncontrollable hypotension, severe bradycardia and hypocardia etc.[13, 14], especially when Dexmedetomidine is used via continuous epidural anesthesia, the risk of its complications increased[15]. Midazolam, belongs to Midazolam benzodiazepines[16' 17], is widely used for anesthesia, procedural sedation, sleep disturbance and severe agitation[18]. It represses the excitatory response via combined with $Y^{-}$ aminobutyric acid receptors and shows similar pharmacological effects as other benzodiazepines[19. 20]. Studies have shown that Midazolam exerts fast anesthetic effect within 5 minutes after intramuscular injection[21], but it may cause loss of airway reflex, respiratory depression and even apnea during anesthesia etc.

The elderly population is at risk of serious complications during the operation and needs special care[22]. Due to the aging population and the advancement of operating technology, more and more elderly people received anesthesia intervention procedures. Although age is an independent risk factor for postoperative mortality and postoperative complications (including cognitive impairment), appropriate anesthesia techniques and drugs can effectively reduce these risks. Therefore, it is necessary to compare safety on effective narcotic sedatives for the elderly. Dexmedetomidine and Midazolam have not yet reached a reliable and consistent conclusion in the sedation of elderly patients. Although a metaanalysis related to anesthesia in children has confirmed that Dexmedetomidine is safer and more effective than Midazolam in terms of sedation and reduction of postoperative complications[23-25]. However, there still lacks comparison on the safety and efficacy between Dexmedetomidine and Midazolam in elderly surgery due to significant heterogeneities between the clinical RCTs, resulting in inconsistent and controversial conclusions. Some studies also reported that there is no significant difference in the sedation between Dexmedetomidine and Midazolam[26-28], thus, the conclusions of these researches have been questioned to a certain extent.

This study is designed to compare the safety and sedative effects between Dexmedetomidine and Midazolam in elderly patients with surgery. In order to minimize the heterogeneity and bias, we will select RCTs addressing the safety and sedative effects of Dexmedetomidine and Midazolam in elderly patients. Systematic reviews and meta-analysis represent an appropriate design to elucidate the clinical usage and efficacy of Dexmedetomidine and Midazolam in previous RCTs, providing clinicians and policymakers with an overall assessment of the evidence on the safety and sedative effects between Dexmedetomidine and Midazolam, which is necessary for both practitioners and elderly patients.

Page 2/10 


\section{Methods}

\section{Study design and Objectives}

The program is designed according to the preferred reporting project guidelines for systematic reviews and meta-analysis programs (PRISMA-P) [29]. It has been registered in the PROSPERO database (CRD42021221897). It aims to provide a systematic review on the effect between Dexmedetomidine and Midazolam in elderly patients. Additionally, meta-analysis of RCTs will be performed in order to assess the safety and efficacy of Dexmedetomidine and Midazolam on primary and secondary outcomes of RCTs.

\section{Search Strategy}

Four English databases (PubMed, Embase, Web of Science, Cochrane Library) and four Chinese databases (Chinese Biomedical Literature Database, Chinese Science and Technology Journals VIP Database, China national knowledge infrastructure (CNKI), Wan-fang Database) were searched for literatures published between October 2001 and May 2021. Only clinical randomized controlled trials (RCTs) published in English or Chinese were included. The search strategy will be conducted according to the Participants, Intervention, Comparison, Outcomes and Studies (PICOS) components (Table 1), The complete search strategy used a combination of PICOS framework, Search strategy in PubMed was seen in Table 2. Any differences can be resolved through discussion with other reviewers (ZW, DBH).

Table 1

Participants, Intervention, Comparison Outcomes and Studies (PICOS) framework.

\begin{tabular}{|c|c|}
\hline PICOS & Searched items \\
\hline \multicolumn{2}{|l|}{$\begin{array}{l}\text { Patients (a } \\
+\mathrm{b})\end{array}$} \\
\hline a & The elderly; The old man; The old patient; The elderly patients; The aged; \\
\hline b & $\begin{array}{l}\text { Intraoperative sedation; Sedation; Surgery sedation, Surgical sedation; Anesthesia and analgesia; Anesthesia sedation; } \\
\text { Intraoperative anesthesia; Surgery anesthesia; Surgical anesthesia }\end{array}$ \\
\hline Intervention & Dexmedetomidine, Dexmedetomidine Hydrochloride, Hydrochloride Dexmedetomidine \\
\hline Comparison & Midazolam, Benzodiazepine, Compounds, Benzodiazepine, Benzodiazepinones, devazepide \\
\hline \multicolumn{2}{|l|}{ Outcome } \\
\hline $\begin{array}{l}\text { Primary } \\
\text { outcomes }\end{array}$ & Hemodynamic changes (MAP, HR, SBP, DBP, RR, SPO2), Sedative effect (OAA/S, BIS) and Cognitive function (MMSE, POCD) \\
\hline $\begin{array}{l}\text { Secondary } \\
\text { outcomes }\end{array}$ & $\begin{array}{l}\text { Analgesic effect (RASS, VAS), Surgical characteristics (operation time, the volume of intraoperative blood loss, time of } \\
\text { extubation), Complications or adverse reactions (agitation, hypotension, bradycardia, respiratory depression, postoperative } \\
\text { nausea and vomiting, bradycardia and shivering) }\end{array}$ \\
\hline Studies & $\begin{array}{l}\text { Random control study, Control study, Random control trial, Clinical control trial, Clinical trial, Randomized control trial, } \\
\text { Randomized controlled trial }\end{array}$ \\
\hline
\end{tabular}


Table 2

Search strategy of PubMed for randomized control trials

\begin{tabular}{|c|c|}
\hline \multicolumn{2}{|c|}{ Searched strategy in PubMed } \\
\hline \multicolumn{2}{|c|}{ Block 1: The elderly } \\
\hline \#1 & "The elderly" [tiab] \\
\hline \#2 & "The old man" [tiab] \\
\hline \#3 & "The old patient" [tiab] OR "The elderly patients" [tiab] \\
\hline \#4 & "The aged" [tiab] OR “The greybeard" [tiab] \\
\hline \#5 & \#1 OR \#2 OR \#3 OR \#4 \\
\hline \multicolumn{2}{|c|}{ Block 2: Intraoperative sedation } \\
\hline \#6 & "Intraoperative sedation" [Mesh] OR “Intraoperative anesthesia” [Mesh] OR “Intraoperative analgesia” [Mesh] \\
\hline \#7 & "Surgical sedation" [tiab] \\
\hline \#8 & "Surgical anesthesia" [tiab] OR "Surgical analgesia" [tiab] \\
\hline \#9 & \#6 OR \#7 OR \#8 \\
\hline \multicolumn{2}{|c|}{ Block 3: Participants } \\
\hline \#10 & \#5 AND \#9 \\
\hline \multicolumn{2}{|c|}{ Block 4: Intervention in experimental group } \\
\hline \#11 & “Dexmedetomidine” [Mesh] \\
\hline \#12 & “Dexmedetomidine hydrochloride” [tiab] OR “Hydrochloride, dexmedetomidine” [tiab] OR “Dexmedetomidine” [tiab] \\
\hline \#13 & \#11 OR \#12 \\
\hline \multicolumn{2}{|c|}{ Block 5: Intervention in control group } \\
\hline \#14 & “Midazolam” [Mesh] \\
\hline \#15 & “Benzodiazepine” [tiab] OR “Benzodiazepines” [tiab] \\
\hline \#16 & "Azepines" [tiab] \\
\hline \#17 & "Hydrochloride, midazolam” [tiab] OR “Midazolam hydrochloride” [tiab] \\
\hline \#18 & “Midazolam maleate" [tiab] OR “Maleate, midazolam” \\
\hline \#19 & \#14 OR \#15 OR \#16 OR \#17 OR \#18 \\
\hline \multicolumn{2}{|c|}{ Block 6: Study type } \\
\hline \#20 & "Randomized controlled trial” [pt] \\
\hline \#21 & “Controlled clinical trial” [pt] \\
\hline \#22 & "Randomized" [tiab] OR “randomly" [tiab] OR "trial” [tiab] OR "groups" [tiab] \\
\hline \#23 & \#20 OR \#21 OR \#22 \\
\hline \multicolumn{2}{|c|}{ Block 6: Final merge } \\
\hline \#24 & \#10 AND \#13 AND \#19 AND \#23 \\
\hline
\end{tabular}

\section{Selection criteria}

Two independent reviewers (HCX, LZJ) screen relevant trials by examining titles and abstracts of the identified studies. Any studies that were not meet with the PICOS framework would be excluded. Full articles will be obtained and checked for extracted details (LYX, LDL). Any reason for extraction would be recorded. Possible divergency will be resolved by discussion or a third reviewer (DBH). The selection process will be presented in a PRISMA flow diagram (Fig. 1).

\section{Eligibility criteria}


Patients with surgery age more than 60 or older and receive anesthetics intervention, whose ASA status have been identified as I-III. Regardless of sex, region, race, and the type of surgery.

\section{Types of intervention in the experimental group and control group}

Patients in the experimental group and control group received Dexmedetomidine or Midazolam respectively, with or without conventional treatment and combination with use of other anesthetics.

\section{Type of included indicators or outcomes}

The primary results contain hemodynamic changes (MAP, HR, SBP, DBP, SPO2), sedative effect (OAA/S, BIS) and cognitive function (MMSE, POCD). The secondary results include analgesic effect (RASS, VAS), surgical characteristics (operation time, the volume of intraoperative blood loss, and extubation time), complications or adverse reactions (hypotension, agitation, postoperative nausea and vomiting, bradycardia, respiratory depression, delirium, and shivering).

\section{Data extraction and risk of bias assessment}

We will extract each qualified data. The differences will be settled through discussion with a third author (ZW). Data were extracted including year of publication, author's name, study type, randomized methodology, and basic characteristics of participants (ASA classification, mean age, sample size, type of surgery, body mass index/weight, duration of anesthesia, duration of surgery, medication use, and outcomes) (Table 3 - 1\&2). Cochrane collaborative tool is used to assess the risk and quality of bias for each included trial, and any differences will be adjusted by the reviewers (ZW, DBH). The Cochrane Collaborative Tool consists of seven quality items: random sequence generation, allocation hiding, blinding of participants and personnel, blinding of result evaluation, incomplete result data, selective reporting and other deviations. Each item is classified as low, unclear or high risk of bias.

\section{Data analysis}

RevMan (version 5.4.1) software is used to complete the meta-analysis. For dichotomous results, odds ratio (ORs) and corresponding $95 \%$ confidence intervals $(95 \% \mathrm{Cl})$ are calculated for comparison. For continuous data, standard mean difference (SMD) and corresponding $95 \% \mathrm{Cl}$ are calculated for comparison. $P$ values less than 0.05 are considered statistically significant. Quantitative calculation of heterogeneity is evaluated using $P^{2}$ statistics. $P^{2}$ statistics are considered to represent the heterogeneity of no (0\%), low (0\%-25\%), medium (25\%-75\%) and high (75\%-100\%) possibility [19]. The random effects model would be adopted if medium or high heterogeneity existed between the studies.

\section{Sensitivity analysis and Subgroup analysis}

When it occurs relatively high heterogeneity of the results, sensitivity analysis is performed to explore the source of high heterogeneity in order to enhance the credibility of the results. Any possibility (methodological quality, heterogeneity, studies quality, surgery duration, number of samples etc.) resulted in high heterogeneity will be considered for subgroup analysis.

\section{Potential publication and reporting bias analysis}

After preliminary meta-analysis, potential publication bias is assessed visually using funnel plots, Egg regression and Begg test will be utilized to detect the funnel plot asymmetry.

Table 3-1

Characteristics of included RCTs assessing effect of Dexmedetomidine and Midazolam for elderly surgery patients (eg.)

\begin{tabular}{|c|c|c|c|c|c|c|c|c|c|}
\hline \multirow[t]{2}{*}{ NO. } & \multirow[t]{2}{*}{ Author(year) } & \multirow[t]{2}{*}{ Type of study (random method) } & \multirow[t]{2}{*}{$\begin{array}{l}\text { ASA } \\
\text { status }\end{array}$} & \multicolumn{2}{|c|}{$\begin{array}{l}\text { Sample size } \\
(\mathrm{M} / \mathrm{F})\end{array}$} & \multicolumn{2}{|c|}{ Age(year) } & \multicolumn{2}{|c|}{ BMI/Weight(kg) } \\
\hline & & & & D & $\mathbf{M}$ & D & M & D & M \\
\hline 1 & $\begin{array}{l}\text { Wenfei Tan } \\
(2016)\end{array}$ & $\begin{array}{l}\text { double blind-RCT (sealed } \\
\text { envelopes) }\end{array}$ & $\mid-I I I$ & 22 & 22 & $\begin{array}{l}70.1 \pm \\
7.0\end{array}$ & $\begin{array}{l}73.1 \pm \\
9.3\end{array}$ & $\begin{array}{l}23.5 \pm \\
3.9\end{array}$ & $\begin{array}{l}22.9 \pm \\
2.3\end{array}$ \\
\hline \multicolumn{10}{|l|}{2} \\
\hline \multicolumn{10}{|l|}{3} \\
\hline \multicolumn{10}{|l|}{...... } \\
\hline \multicolumn{10}{|l|}{$\ldots \ldots$} \\
\hline
\end{tabular}


Table 3-2

Characteristics of included RCTs assessing effect of Dexmedetomidine and Midazolam for elderly surgery patients (eg.)

\begin{tabular}{|c|c|c|c|c|c|c|c|c|c|c|}
\hline \multirow[t]{2}{*}{ NO. } & \multirow[t]{2}{*}{ Author(year) } & \multicolumn{2}{|c|}{$\begin{array}{l}\text { Anesthesia } \\
\text { duration }\end{array}$} & \multicolumn{2}{|c|}{$\begin{array}{l}\text { Operation } \\
\text { duration }\end{array}$} & \multicolumn{2}{|c|}{ Dosage and Usage of D } & \multicolumn{2}{|c|}{ Dosage and Usage of $M$} & \multirow[t]{2}{*}{$\begin{array}{l}\text { Type of } \\
\text { Disease }\end{array}$} \\
\hline & & $\mathrm{D}(\mathrm{min})$ & $M(\min )$ & $\mathrm{D}(\mathrm{min})$ & $M(\min )$ & $\begin{array}{l}\text { Anesthesia } \\
\text { induction }\end{array}$ & $\begin{array}{l}\text { Anesthesia } \\
\text { maintenance }\end{array}$ & $\begin{array}{l}\text { Anesthesia } \\
\text { induction }\end{array}$ & $\begin{array}{l}\text { Anesthesia } \\
\text { maintenance }\end{array}$ & \\
\hline 1 & $\begin{array}{l}\text { Wenfei Tan } \\
(2016)\end{array}$ & $\begin{array}{l}91.9 \pm \\
28.0\end{array}$ & $\begin{array}{l}99.9 \pm \\
46.3\end{array}$ & $\begin{array}{l}69.9 \pm \\
23.4\end{array}$ & $\begin{array}{l}78.2 \pm \\
46.9\end{array}$ & $\begin{array}{l}1 \mathrm{ug} / \mathrm{kg} \text { for } \\
10 \mathrm{~min}\end{array}$ & $12 \mathrm{ug} /(\mathrm{kg} \cdot \mathrm{h})$ & $\begin{array}{l}22.5 \mathrm{ug} /(\mathrm{kg} \cdot \mathrm{h}) \\
\text { for } 10 \mathrm{~min}\end{array}$ & $45 \mathrm{ug} /(\mathrm{kg} \cdot \mathrm{h})$ & $\begin{array}{l}\text { elective } \\
\text { transurethral } \\
\text { prostatic } \\
\text { resection }\end{array}$ \\
\hline
\end{tabular}

2

3

…...

Note: ND: Not described in the study; NOT: No dosage and usage of anesthesia; D: Dexmedetomidine; M: Midazolam.

\section{Test sequential analysis (TSA)}

For further illustrating and confirming the reliability of final results, trial sequential analysis is performed on the analyzed outcomes via TSA software (version 0.9.5.10 Beta; Copenhagen Trial Unit, Center for Clinical Intervention Research, Rigshospitalet, Copenhagen, Denmark), aiming to rule out the possibility of false positives and confirm firm evidence of final results.

\section{Rating quality of evidence and strength of recommendations (GRADE)}

Finally, we will conduct evaluation on the quality of evidence regarding to the main positive outcomes using the GRADE Profiler 3.6.1 evaluation tool. Briefly, the quality of the evidence is divided into four levels: high, medium, low and very low, and strength of recommendations are divided into strong recommendation and weak recommendation. RCT trials are regarded as high quality of evidence, and then the evidence quality level of the outcomes are assessed from the five downgrading factors of research limitations, inconsistency, indirectness, imprecision, and publication bias.

\section{Discussion}

Secure and effective anesthetics effectively reduce the occurrence of postoperative complications, and improve peri-operative cognitive dysfunction or intra-operative awareness, providing clinicians with better surgery procedures[30]. However, appropriate anesthetic for elderly patients with surgery is still a serious challenge for clinicians[31]. Up to date, there is not enough evidence to determine a better anesthesia plan for elderly patients[32].

Various drugs including ketamine, propofol, benzodiazepines, fentanyl, etomidate and Dexmedetomidine etc. exert intraoperative sedation. Midazolam and Dexmedetomidine is both applied for anesthesia and sedation in the elderly[33]. However, studies have indicated that Midazolam led to cognitive impairment, postoperative or delirium in the elderly[34]. Although Dexmedetomidine does not directly damage the respiratory system and rarely causes apnea, it has been suggested that it cause impaired to the respiratory system response to hypoxia and hypercapnia[35], it can also lead to hemodynamic effects such as hypertension, hypotension and bradycardia. Whereas, in some clinical studies, their conclusions are inconsistent and suspicious due to their heterogeneity in research methods[36, 37]. Up to date, it still lacks comprehensive acknowledgement on the safety and efficacy of Dexmedetomidine and Midazolam.

In conclusion, systematically evaluation on the efficacy and safety of Dexmedetomidine and Midazolam for intraoperative sedation in elderly patients can provide the anesthesiologist with insight to the appropriate anesthetic choice, aiming to reducing the potential postoperative complications of elderly patients[38]. This protocol maximizes the extraction of relevant information with a clear and structured procedure, however, we also acknowledged several limitations existing in this systematic review and meta-analysis. The assessment methods differed in different included studies may results in medium or high heterogeneity. Some of the included studies are of low quality. Moreover, only studies published in English and Chines will be included. Therefore, we will continue to pay attention to update our conclusions in future.

\section{Abbreviations}




\begin{tabular}{|llll|}
\hline Abbreviations & Full name & Abbreviations & Full name \\
\hline RCTs & Randomized controlled trials & MAP & Mean arterial pressure \\
\hline RASS & Richmond Agitation-Sedation Scale & SBP & Systolic blood pressure \\
\hline MMSE & Mini-mental State Examination & DBP & Diastolic blood pressure \\
\hline PICOS & Patients, intervention, control, outcomes, study & OAA/S & Observer assessment of alertness/sedation scale \\
\hline BIS & Bispectral index & POCD & postoperative cognitive dysfunction \\
\hline VAS & Visual Analogue Scale/Score & SPO2 & Saturation of Pulse Oxygen \\
\hline HR & Heart rate & OP & Odds ratio \\
\hline ASA & American Society of Anesthesiologists & $95 \% \mathrm{Cl}$ & 95\% confidence intervals \\
\hline SMD & Standard mean difference & TSA & Test sequential analysis \\
\hline
\end{tabular}

\section{Declarations}

\section{Ethics approval and consent to participate}

The study is a meta-analytic review. All data included in this study will be extracted from published articles and not involve personal privacy of patients, thus, ethical approval is not applicable.

\section{Consent for publication}

Not applicable.

\section{Availability of data and materials}

The datasets used and/or analysed during the current study are available from the corresponding author on reasonable request.

\section{Competing interests}

There has no competing interests between authors.

\section{Funding}

This work was supported by the special foundation of Guangzhou Key Laboratory (No. 202002010004); Science and Technology Planning Project of Guangdong Province (No. 2017B030314166), Guangdong Provincial Hospital of Chinese Medicine Science and Technology Research Program (No. YN2019MJ11) and Secondary Development Project of Guangdong Provincial Pharmaceutical Chinese medicine (No. 20170410).

\section{Authors' contributions}

DBH and ZW are contributed to the conception of the study, this manuscript was drafted by HCX and LZJ, it was revised by LYX. The search strategy was developed by all authors and will be conducted by HCX and LZJ, LYX and LDL will extract data from included studies and assess the risk of bias. HCX and HMH will conduct and finish the data synthesis. DBH and ZW will arbitrate in cases of disagreement and ensure no errors occur during the study. All authors have approved the publication of the protocol.

\section{Acknowledgements}

Not applicable.

\section{References}

1. E. Jaul, J. Barron. Age-Related Diseases and Clinical and Public Health Implications for the 85 Years Old and Over Population. Front Public Health.2017; 5 335.doi:10.3389/fpubh.2017.00335.

2. E. Montecino-Rodriguez, B. Berent-Maoz, K. Dorshkind. Causes, consequences, and reversal of immune system aging. Journal of Clinical Investigation.2013; 123(3), 958-965.doi:10.1172/JCI64096.

3. S. Aucoin, D. I. Mclsaac. Emergency General Surgery in Older Adults: A Review. Anesthesiol Clin.2019; 37(3), 493505.doi:10.1016/j.anclin.2019.04.008. 
4. F. C. Ko. Preoperative Frailty Evaluation: A Promising Risk-stratification Tool in Older Adults Undergoing General Surgery. Clinical Therapeutics.2019; 41(3), 387-399.doi:10.1016/j.clinthera.2019.01.014.

5. Y. Jauhari, M. R. Gannon, D. Dodwell, K. Horgan, C. Tsang, K. Clements, J. Medina, S. Tang, R. Pettengell, D. A. Cromwell. Addressing frailty in patients with breast cancer: A review of the literature. Eur J Surg Oncol.2020; 46(1), 24-32.doi:10.1016/j.ejso.2019.08.011.

6. J. S. Partridge, D. Harari, J. K. Dhesi. Frailty in the older surgical patient: a review. Age and Ageing.2012; 41(2), 142147.doi:10.1093/ageing/afr182.

7. C. McPherson, C. M. Ortinau, Z. Vesoulis. Practical approaches to sedation and analgesia in the newborn. Journal of Perinatology.2021; 41(3), 383-395.doi:10.1038/s41372-020-00878-7.

8. K. J. Anand, R. W. Hall. Pharmacological therapy for analgesia and sedation in the newborn. Arch Dis Child Fetal Neonatal Ed.2006; 91(6), F448-F453.doi:10.1136/adc.2005.082263.

9. J. Afonso, F. Reis. Dexmedetomidine: current role in anesthesia and intensive care. Revista Brasileira De Anestesiologia.2012; 62(1), 118133.doi:10.1016/S0034-7094(12)70110-1.

10. S. Naaz, E. Ozair. Dexmedetomidine in current anaesthesia practice- a review. J Clin Diagn Res.2014; 8(10), E1E4.doi:10.7860/JCDR/2014/9624.4946.

11. O. M. Romera, J. C. Chamorro, V. I. Lipperheide, S. I. Fernandez. Indications of dexmedetomidine in the current sedoanalgesia tendencies in critical patients. Medicina Intensiva.2014; 38(1), 41-48.doi:10.1016/j.medin.2013.03.008.

12. R. Wiatrowski. Current State of Pain Treatment: Does Dexmedetomidine Have a Role to Play? AANA J.2021; 89(1), 77-86.

13. A. T. Gerlach, C. V. Murphy. Dexmedetomidine-associated bradycardia progressing to pulseless electrical activity: case report and review of the literature. Pharmacotherapy.2009; 29(12), 1492.doi:10.1592/phco.29.12.1492.

14. A. Aikaterini, D. loannis, G. Dimitrios, S. Konstantinos, G. Vasilios, P. George. Bradycardia Leading to Asystole Following Dexmedetomidine Infusion during Cataract Surgery: Dexmedetomidine-Induced Asystole for Cataract Surgery. Case Rep Anesthesiol.2018; 2018 2896032.doi:10.1155/2018/2896032.

15. J. Mantz, J. Josserand, S. Hamada. Dexmedetomidine: new insights. Eur J Anaesthesiol.2011; 28(1), 36.doi:10.1097/EJA.0b013e32833e266d.

16. C. Gade, E. Sverrisdottir, K. Dalhoff, J. Sonne, M. O. Johansen, H. R. Christensen, J. Burhenne, G. Mikus, J. C. Holm, T. M. Lund, H. Holst. Midazolam Pharmacokinetics in Obese and Non-obese Children and Adolescents. Clinical Pharmacokinetics.2020; 59(5), 643654.doi:10.1007/s40262-019-00838-1.

17. U. Klotz. Pharmacokinetics and drug metabolism in the elderly. Drug Metabolism Reviews.2009; 41(2), 6776.doi:10.1080/03602530902722679.

18. T. Azeem, N. E. Yosif, A. M. Alansary, I. M. Esmat, A. K. Mohamed. Dexmedetomidine vs morphine and midazolam in the prevention and treatment of delirium after adult cardiac surgery; a randomized, double-blinded clinical trial. Saudi J Anaesth.2018; 12(2), 190197.doi:10.4103/sja.SJA_303_17.

19. P. Ashley, P. Anand, K. Andersson. Best clinical practice guidance for conscious sedation of children undergoing dental treatment: an EAPD policy document. Eur Arch Paediatr Dent.2021;doi:10.1007/s40368-021-00660-z.

20. A. Kupietzky, M. I. Houpt. Midazolam: a review of its use for conscious sedation of children. Pediatr Dent.1993; $15(4), 237-241$.

21. J. W. Taylor, K. B. Simon. Possible intramuscular midazolam-associated cardiorespiratory arrest and death. DICP.1990; 24(7-8), 695697.doi:10.1177/106002809002400707.

22. A. Meziere, E. Paillaud, B. Plaud. Anesthesia in the elderly. Presse Medicale.2013; 42(2), 197-201.doi:10.1016/j.lpm.2012.07.040.

23. L. Pasin, D. Febres, V. Testa, E. Frati, G. Borghi, G. Landoni, A. Zangrillo. Dexmedetomidine vs midazolam as preanesthetic medication in children: a meta-analysis of randomized controlled trials. Paediatr Anaesth.2015; 25(5), 468-476.doi:10.1111/pan.12587.

24. Y. Sun, Y. Lu, Y. Huang, H. Jiang. Is dexmedetomidine superior to midazolam as a premedication in children? A meta-analysis of randomized controlled trials. Paediatr Anaesth.2014; 24(8), 863-874.doi:10.1111/pan.12391.

25. B. Lang, L. Zhang, W. Zhang, Y. Lin, Y. Fu, S. Chen. A comparative evaluation of dexmedetomidine and midazolam in pediatric sedation: A meta-analysis of randomized controlled trials with trial sequential analysis. CNS Neuroscience \& Therapeutics.2020; 26(8), 862875.doi:10.1111/cns.13377.

26. M. Sathyamoorthy, T. B. Hamilton, G. Wilson, R. Talluri, L. Fawad, B. Adamiak, C. Wallace, I. Borissova, C. Heard. Pre-medication before dental procedures: A randomized controlled study comparing intranasal dexmedetomidine with oral midazolam. Acta Anaesthesiol Scand.2019; 63(9), 1162-1168.doi:10.1111/aas.13425.

27. H. S. Abdel-Ghaffar, S. M. Kamal, S. F. El, S. A. Mohamed. Comparison of nebulised dexmedetomidine, ketamine, or midazolam for premedication in preschool children undergoing bone marrow biopsy. Br J Anaesth.2018; 121(2), 445-452.doi:10.1016/j.bja.2018.03.039.

28. B. Sajid, T. Mohamed, M. Jumaila. A comparison of oral dexmedetomidine and oral midazolam as premedicants in children. $J$ Anaesthesiol Clin Pharmacol.2019; 35(1), 36-40.doi:10.4103/joacp.JOACP_20_18.

Page 8/10 
29. D. Moher, L. Shamseer, M. Clarke, D. Ghersi, A. Liberati, M. Petticrew, P. Shekelle, L. A. Stewart. Preferred reporting items for systematic review and meta-analysis protocols (PRISMA-P) 2015 statement. Syst Rev.2015; 4 1.doi:10.1186/2046-4053-4-1.

30. L. D. Gray, C. Morris. The principles and conduct of anaesthesia for emergency surgery. Anaesthesia.2013; 68 Suppl $114-$ 29.doi:10.1111/anae.12057.

31. S. M. Kruijt, N. A. Bakker, A. R. Absalom. Pharmacology in the elderly and newer anaesthesia drugs. Best Pract Res Clin Anaesthesiol.2011; 25(3), 355-365.doi:10.1016/j.bpa.2011.06.002.

32. B. G. Lim, I. O. Lee. Anesthetic management of geriatric patients. Korean J Anesthesiol.2020; 73(1), 8-29.doi:10.4097/kja.19391.

33. C. R. Barends, A. Absalom, B. van Minnen, A. Vissink, A. Visser. Dexmedetomidine versus Midazolam in Procedural Sedation. A Systematic Review of Efficacy and Safety. PLoS One.2017; 12(1), e169525.doi:10.1371/journal.pone.0169525.

34. T. L. Griebling. Re: American Geriatrics Society 2019 Updated AGS Beers Criteria(R) for Potentially Inappropriate Medication Use in Older Adults. J Urol.2019; 202(3), 438.doi:10.1097/JU.0000000000000409.

35. J. M. Constantin, A. Momon, J. Mantz, J. F. Payen, B. De Jonghe, S. Perbet, S. Cayot, G. Chanques, B. Perreira. Efficacy and safety of sedation with dexmedetomidine in critical care patients: a meta-analysis of randomized controlled trials. Anaesth Crit Care Pain Med.2016; 35(1), 715.doi:10.1016/j.accpm.2015.06.012.

36. S. M. White, N. B. Foss, R. Griffiths. Anaesthetic aspects in the treatment of fragility fracture patients. Injury-International Journal of the Care of the Injured.2018; 49(8), 1403-1408.doi:10.1016/j.injury.2018.06.027.

37. S. M. White, F. Altermatt, J. Barry, B. Ben-David, M. Coburn, F. Coluzzi, M. Degoli, D. Dillane, N. B. Foss, A. Gelmanas, R. Griffiths, G. Karpetas, J. H. Kim, M. Kluger, P. W. Lau, I. Matot, M. McBrien, S. McManus, L. F. Montoya-Pelaez, I. K. Moppett, M. Parker, O. Porrill, R. D. Sanders, C. Shelton, F. Sieber, A. Trikha, X. Xuebing. International Fragility Fracture Network Delphi consensus statement on the principles of anaesthesia for patients with hip fracture. Anaesthesia.2018; 73(7), 863-874.doi:10.1111/anae.14225.

38. B. G. Lim, I. O. Lee. Anesthetic management of geriatric patients. Korean J Anesthesiol.2020; 73(1), 8-29.doi:10.4097/kja.19391.

\section{Figures}




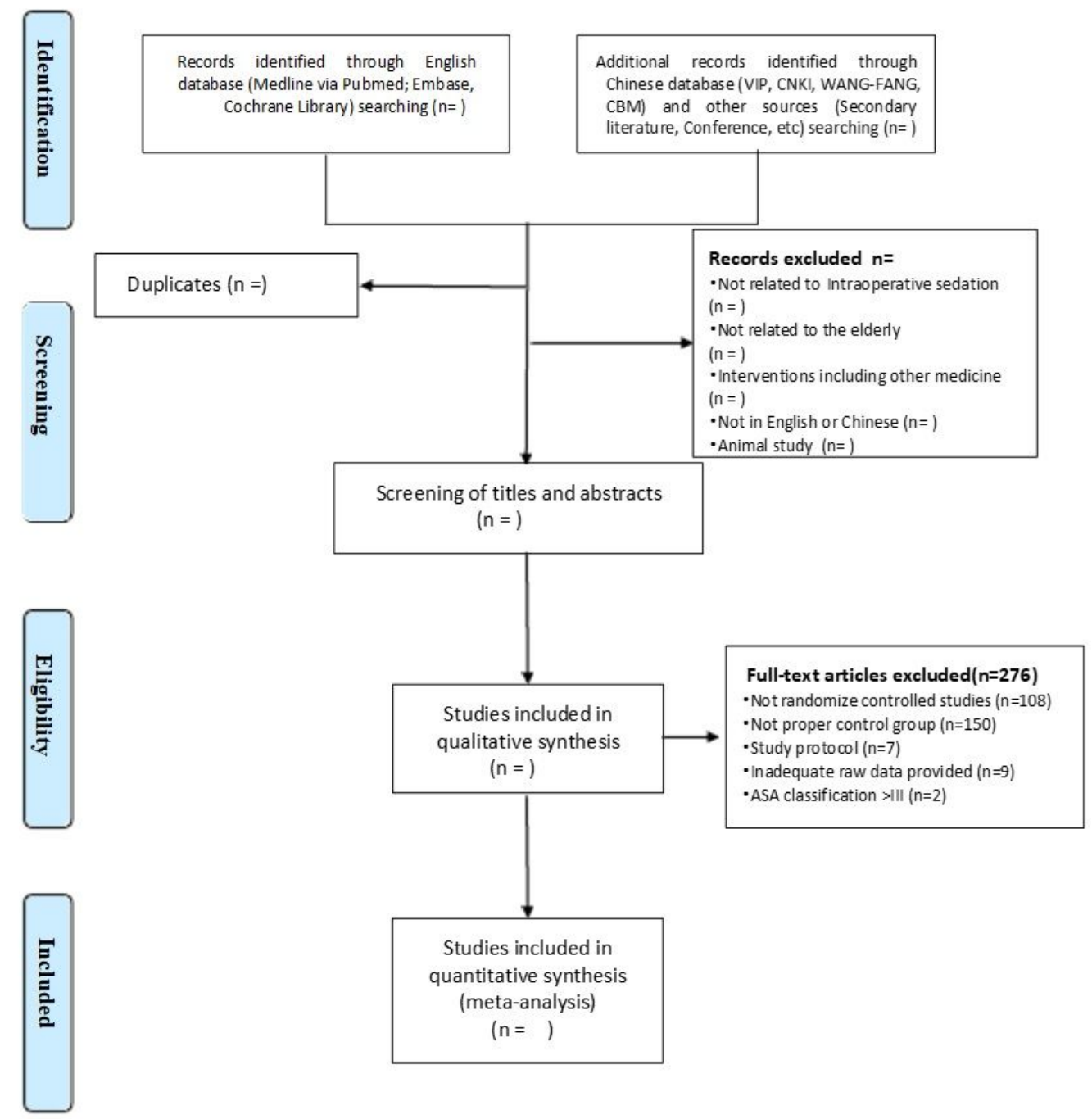

Figure 1

PRISMA flow chart depicting studies included in the systematic review and meta-analysis.

\section{Supplementary Files}

This is a list of supplementary files associated with this preprint. Click to download.

- PRISMAPchecklist.docx 